\title{
A Fuzzy Approach for Overcurrent Relays Simulation
}

\author{
Hossein Askarian Abyaneh ${ }^{1}$, Hossein Kazemi Karegar ${ }^{1}$, and Majid Al-Dabbagh ${ }^{2}$ \\ ${ }^{1}$ Department of Electrical Engineering , Amir Kabir University of Technology, Hafez Ave, No 424 \\ P.O. Box 15875-4413, Tehran, Iran \\ haskarian@yahoo.com \\ h_kazemi_ir@yahoo.com \\ ${ }^{2}$ School of Electrical Engineering and Computer System of RMIT University, Victoria 3001 \\ Melbourne, Australia \\ majid@rmit.edu.au
}

\begin{abstract}
Accurate models for Overcurrent relays (OC) with inverse time characteristics have an important role for efficient coordination of power system protection devices. This paper proposes a new method for modelling of OC relays based on fuzzy logic. The new model is more accurate than traditional models and has been validated by comparing it with the linear and nonlinear analytical models.
\end{abstract}

\section{Introduction}

Due to unforseen load expansion, many power systems are operated close to their design limits, therefore it is necessary to revise modelling of relays under these conditions [1]. OC relay models are defined in various ways. Time-Current (TC) curves of an OC relay is the most commonly used method [2]. There are two main methods for representing an $\mathrm{OC}$ relay i.e., by using digital computers: software models and direct data storage. Software models of OC relay characteristics play a major role in coordinating protection schemes in power systems [3].

A comprehensive review of computer representation of $\mathrm{OC}$ relays has been made in reference [4]. In this review, it is stated that Sachdev models are simple and useful polynomials for modelling OC relays for coordination purposes. It should be noted that any microprocessor relay abiding to the IEEE Std. C37.112 [5], should follow in this representation the equations provided by their standard. Furthermore in this context, the time dial setting provided by this standard is linear.

An alternative method for the representation of OC relays is based on direct data storage techniques. These techniques store data in memory of the computers for different TDS/TMS and then select operating points of a relay based on the stored data for different TDS/TMS. If the selected operating point does not match with one of the stored values an interpolation is necessary to determine the corresponding time. Therefore, this method requires storing large amount of data.

In this paper a new model based on fuzzy logic and is more accurate than analytical models and does not need look up tables is presented. The new methodology employed gives a more accurate model, compared with the model introduced in [6]. 


\section{The New Method}

The proposed fuzzy model is based on finding a simple mathematical equation with a fuzzy correction coefficient to calculate the operating time of OC relays.

In Fig. $1,\left(t_{1}, I_{1}\right),\left(t_{2}, I_{2}\right)$ and $\left(t_{3}, I_{3}\right)$, sampled data are given is the operating time of OC relay for a given $I^{*}$. The simplest equation for fitting two points on a curve is the straight line equation. This mathematical equation does not need any complicated curve fitting technique. In reality, two adjacent points on OC relay characteristics are connected as a line. Therefore, the proposed fuzzy logic model finds a fuzzy correction coefficient to simulate the curve of the OC relay under consideration.

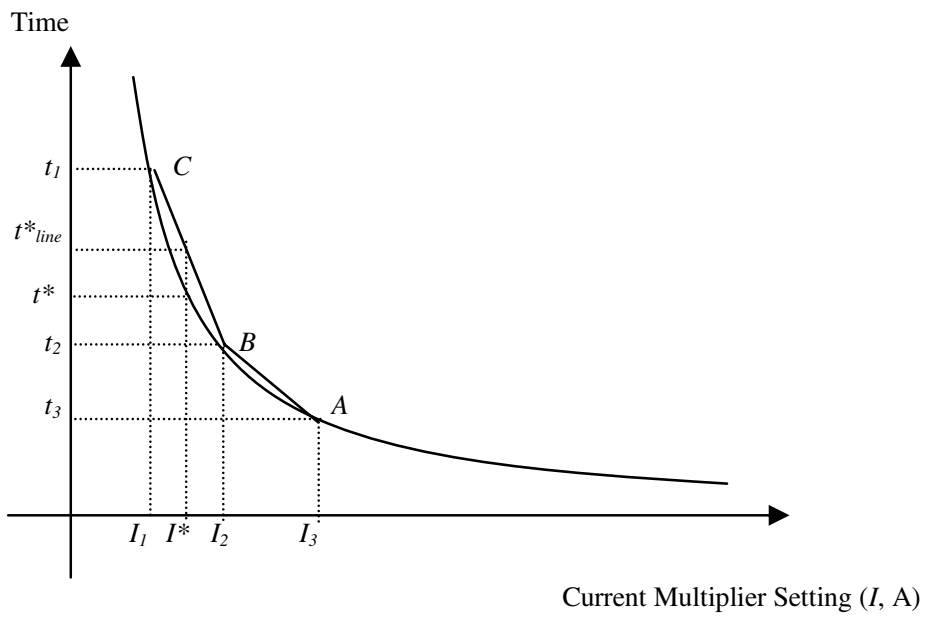

Fig. 1. A TC curve of an overcurrent relay

If the two points $\left(t_{1}, I_{1}\right)$ and $\left(t_{2}, I_{2}\right)$ of Fig. 1 are the considered sampled data, then the operation time associated with $\mathrm{I}^{*}$ can be calculated by As follows:

$$
t^{*} \text { line }=\left(t_{2}-t_{1}\right) \cdot\left(I^{*}-I_{1}\right) /\left(I_{2}-I_{1}\right)+t_{1}
$$

The calculated operation time according to equation (1) is $t *$ Line which differes from actual one, i.e. $t^{*}$. Therefore, a correction factor must be added to equation (1). The variation of correction factor, $r$, is determined using fuzzy logic technique. The equation with correction factor is given as:

$$
t^{*}=r .\left(t_{2}-t_{1}\right) \cdot\left(I^{*}-I_{1}\right) /\left(I_{2}-I_{1}\right)+t_{1}
$$

The fuzzy correction coefficient $r$ varies between 0 and 1 when the location of $I^{*}$ changes on the current multiplier setting axis of TC curves. The values of $I^{*}$ and the 
slope $(s)$ of the line between adjacent data, play an important role in calculating the variation of $r$. For example, when $I^{*}$ gets large and goes near the tale of the TC curve, and the slope gets smaller. Subsequently, the curve between two neighbouring data is close to a direct line. Therefore, the value of $r$ increases and approaches a value close to one.

Equations (3) and (4) describe the calculation of $r$ and $s$ for the sampled data.

$$
\begin{gathered}
r_{i}=\left[\left(I_{i+2}-I_{i}\right) .\left(t_{i+1}-t_{i}\right)\right] /\left[\left(t_{i+2}-t_{i}\right) .\left(I_{i+1}-I_{i}\right)\right] \\
s_{i}=\left(t_{i+1}-t_{i}\right) /\left(I_{i+1}-I_{i}\right)
\end{gathered}
$$

For example, in considering Fig. 1, $r_{l}$ can be obtained by Eq. (5).

$$
r_{1}=\left[\left(I_{3}-I_{1}\right) \cdot\left(t_{2}-t_{1}\right)\right] /\left[\left(I_{3}-I_{1}\right) \cdot\left(t_{2}-t_{1}\right)\right]
$$

The membership functions of $I, r$ and $s$ of sampled data are necessary for calculating the value of $r$ for other set of data. It is worth mentioning that the value of $I$ can be obtained from the catalogues of OC relays. For example, $I$ varies from 2 to 30 for RSA20, an electromechanical OC relay. However, the values of $s$ and $r$ must be calculated based on the stored data and then used for the calculation of the membership functions of $r\left(\mu_{r}\right)$ and $s\left(\mu_{s}\right)$. Because the values of $I, r$ and $s$ are positive, only Positive Small (PS), Positive Medium (PM) and Positive Big (PB) are effective.

The membership functions of $I, r$ and $s$ are shown in Fig. 2.

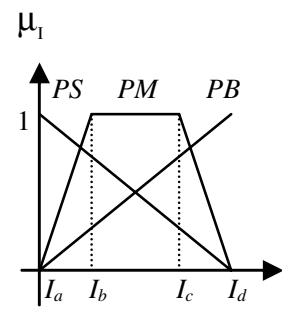

$\mu_{\mathrm{s}}$

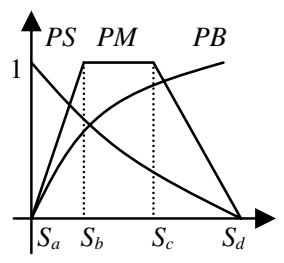

$\mu_{\mathrm{r}}$

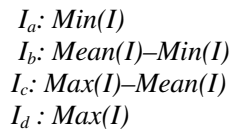

$$
\begin{aligned}
& S_{a}: \operatorname{Min}(S) \\
& S_{b}: \operatorname{Mean}(S)-\operatorname{Min}(S) \\
& S_{c}: \operatorname{Max}(S)-\operatorname{Mean}(S) \\
& S_{d}: \operatorname{Max}(I)
\end{aligned}
$$

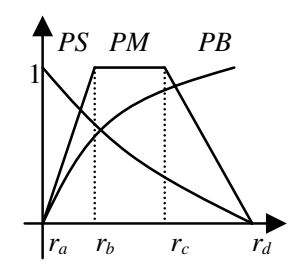

Fig. 2. Membership functions of $I, r$ and $s$

It is found by trial and error that the trapezoid shape for PM produces better results than triangular shape, which is used in reference [6]. This feature is regarded as an advantage in the new fuzzy model, compared with the previous method, 
Determining fuzzy rules are the next step in fuzzy modeling. As can be seen from Fig. 1, when $I$ is small, near pick-up current, and s is large, because TC curve closes to its asymptotic, then the value of $r$ must be selected large, i.e., 1. In other words, TC curves of OC relays near the pick-up currents are very straight like a direct line.

Based on above assumptions, the fuzzy rules for the proposed method are as below:

- If $I$ is small and $s$ is large then $r$ is large.

- If $I$ is small and $s$ is medium then $r$ is medium.

- If $I$ is medium and $s$ is small then $r$ is medium.

- If $I$ is medium and $s$ is medium then $r$ is small.

- If $I$ is medium and $s$ is large then $r$ is medium.

- If $I$ is large and $s$ is medium then $r$ is medium.

- If $I$ is large and $s$ is small then $r$ is large.

The last step in fuzzy modelling is determining the value of $r$. In this paper, the centroid method is used to calculate $r$. Equations (6)-(8) show how to obtain $r$.

$$
\begin{gathered}
a_{i}=\mu_{i} \wedge \mu_{s} \\
\mu_{r}^{\prime}=a_{i} \wedge \mu_{r} \\
r_{i}=\operatorname{centroid}\left(\mu_{r}^{\prime}\right) \\
r=\sum_{1}^{n} a_{i} \cdot r_{i} / \sum_{1}^{n} a_{i}
\end{gathered}
$$

Equation (9) describes the normalized weight method to produce a single output corresponding to $\mathrm{r}[7]$.

\section{Case Study}

Two types of OC relays were used for evaluating the proposed model. The first one was RSA20, an electromechanical OC relay, where its TDS varies from 4 to 20. The second one was CRP9, a very inverse electromechanical OC relay. For both relays, the used sampled data are shown in Table 1 and Table 2 respectively, Additional sampled data which is shown in Tables 3 and 4 is used for testing and comparing the analytical and the proposed models. 
Table 1. Operation time of RSA20 OC relay in ms when TDS $=4,8,14$ and 20

\begin{tabular}{ccccc}
\hline I & TDS=4 & TDS=8 & TDS=14 & TDS=20 \\
& & & & \\
\hline 4 & 4238 & 5690 & 8840 & 11975 \\
5.5 & 2235 & 3255 & 5439 & 7516 \\
7 & 1648 & 2412 & 4012 & 5797 \\
8.5 & 1373 & 2070 & 3522 & 5070 \\
10 & 1240 & 1960 & 3283 & 4702 \\
11.5 & 1155 & 1890 & 3146 & 4525 \\
13 & 1112 & 1825 & 3076 & 4411 \\
14.5 & 1075 & 1778 & 3025 & 4333 \\
16 & 1061 & 1754 & 2993 & 4264 \\
17.5 & 1045 & 1735 & 2954 & 4194 \\
19 & 1028 & 1714 & 2911 & 4126 \\
20.5 & 1017 & 1691 & 2874 & 4083 \\
22 & 1010 & 1659 & 2846 & 4043 \\
23.5 & 1008 & 1636 & 2799 & 4003 \\
25 & 1006 & 1626 & 2771 & 3965 \\
26.5 & 1004 & 1611 & 2747 & 3935 \\
28 & 1002 & 1603 & 2734 & 3915 \\
29.5 & 1000 & 1594 & 2697 & 3893 \\
\hline
\end{tabular}

Table 2. Operation time of CRP9 OC relay in $\mathrm{ms}$ when $\mathrm{TDS}=4,6,7$ and 10

\begin{tabular}{ccccc}
\hline I & TDS=4 & TDS=6 & TDS=7 & TDS=10 \\
\hline 3 & 2451 & 3521 & 4232 & 6495 \\
4 & 1352 & 2107 & 2459 & 3935 \\
5 & 953 & 1527 & 1853 & 2734 \\
6 & 758 & 1243 & 1517 & 2175 \\
7 & 653 & 1054 & 1302 & 1927 \\
8 & 605 & 934 & 1153 & 1754 \\
9 & 552 & 836 & 1051 & 1621 \\
10 & 516 & 742 & 993 & 1523 \\
12 & 459 & 658 & 894 & 1304 \\
14 & 401 & 634 & 794 & 1221 \\
16 & 375 & 618 & 744 & 1123 \\
18 & 359 & 608 & 715 & 1059 \\
20 & 358 & 585 & 669 & 1024 \\
\hline
\end{tabular}


Table 3. Test operation time of RSA20 OC relay in ms when TDS $=4,8,14$ and 20

\begin{tabular}{ccccc}
\hline II & TDS=4 & TDS=8 & TDS=14 & TDS=20 \\
\hline 4.5 & 2816 & 4220 & 6637 & 9097 \\
6 & 1881 & 2751 & 4512 & 6496 \\
7.5 & 1478 & 2196 & 3706 & 5319 \\
9 & 1314 & 2005 & 3373 & 4863 \\
10.5 & 1206 & 1930 & 3206 & 4617 \\
12 & 1128 & 1860 & 3105 & 4461 \\
13.5 & 1096 & 1802 & 3049 & 4370 \\
15 & 1074 & 1765 & 3008 & 4303 \\
16.5 & 1051 & 1743 & 2971 & 4228 \\
18 & 1032 & 1720 & 2934 & 4158 \\
19.5 & 1025 & 1702 & 2894 & 4106 \\
21 & 1015 & 1685 & 2862 & 4066 \\
22.5 & 1009 & 1652 & 2825 & 4023 \\
24 & 1007 & 1634 & 2785 & 3982 \\
25.5 & 1005 & 1616 & 2756 & 3951 \\
27 & 1003 & 1607 & 2743 & 3929 \\
28.5 & 1001 & 1597 & 2718 & 3901 \\
30 & 998 & 1593 & 2689 & 3886 \\
\hline
\end{tabular}

Table 4. Test operation time of CRP9 OC relay in ms when TDS=4, 6, 8 and 10

\begin{tabular}{lcccc}
\hline 1I & TDS=4 & TDS=6 & TDS=8 & TDS=10 \\
\hline 3.5 & 1721 & 2832 & 3365 & 5241 \\
4.5 & 1125 & 1746 & 2123 & 3289 \\
5.5 & 849 & 1369 & 1648 & 2351 \\
6.5 & 695 & 1124 & 1411 & 1986 \\
7.5 & 634 & 1009 & 1213 & 1821 \\
8.5 & 574 & 892 & 1098 & 1698 \\
9.5 & 534 & 776 & 1035 & 1586 \\
11 & 489 & 692 & 939 & 1401 \\
13 & 425 & 642 & 835 & 1248 \\
15 & 386 & 623 & 755 & 1167 \\
17 & 367 & 612 & 732 & 1087 \\
19 & 357 & 593 & 684 & 1048 \\
\hline
\end{tabular}

The sampled data is obtained by performing experimental tests several times using an accurate computerised relay tester for RSA20 and CRP9 relays, to ensure the accuracy of the recorded expanded data. 


\subsection{Fuzzy Model Application}

\subsubsection{Fuzzy Model of RSA20 OC Relay}

The recommended analytical model in reference [4] is selected for comparison between the new and the mathematical model. Due to the progresses in both calculating methods and software packages an equation with more polynomials coefficients is possible and provides more accurate results. MATLAB has provided a useful environment to calculate polynomials coefficients based on advanced non-linear curve fitting techniques [11,12]. Therefore, Eq. (10) with nine coefficients is chosen. It should be noted that when a polynomial equation with more than nine coefficients was used, ill-structure matrices and poor output results were reported by MATLAB. In addition, normalized operation time data were used to improve the accuracy of the obtained results by

$$
t=a_{0}+a_{1} /(I-1)+a_{2} /(I-1)^{2}+. .+a_{8} /(I-1)^{8}
$$

The coefficients of Eq. (10), i.e. $a_{0}$ to $a_{8}$, are shown in Table 5 where data in columns TDS=4, 8 and 20 of Table 1 are selected as input. The first column of Table 5 shows the coefficients of Eq. (10) for the sampled data of TC curve when TDS=6.

Table 5. Polynomial coefficients of RSA20 OC relay when TDS $=4,8$ and 20

\begin{tabular}{cccc}
\hline Coefficient & TDS $=6$ & TDS $=8$ & TDS $=20$ \\
\hline$a_{0}$ & 0.2343 & 0.2386 & 0.2924 \\
$a_{1}$ & -0.1957 & 1.4592 & 1.0945 \\
$a_{2}$ & 6.222 & -9.0501 & -4.7457 \\
$a_{3}$ & -0.0351 & 36.2787 & 26.2756 \\
$a_{4}$ & -2.9201 & 25.0367 & 14.3067 \\
$a_{5}$ & 2.259 & -39.6346 & -33.2044 \\
$a_{6}$ & 11.4195 & -117.835 & -87.3217 \\
$a_{7}$ & 20.7218 & -186.711 & -133.39 \\
$a_{8}$ & 28.6445 & -239.456 & -167.828 \\
\hline
\end{tabular}

By calculating the average error percentage for each curve of RSA20, the two curves illustrating the mean error percentage of the fuzzy and the mathematical ninecoefficient model are shown in Fig. 4 and Fig. 5.

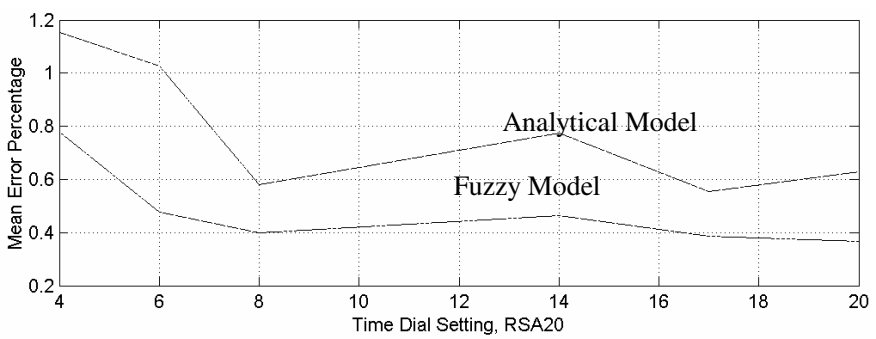

Fig. 4. Average error percentage of fuzzy and Analytical model for RSA20 


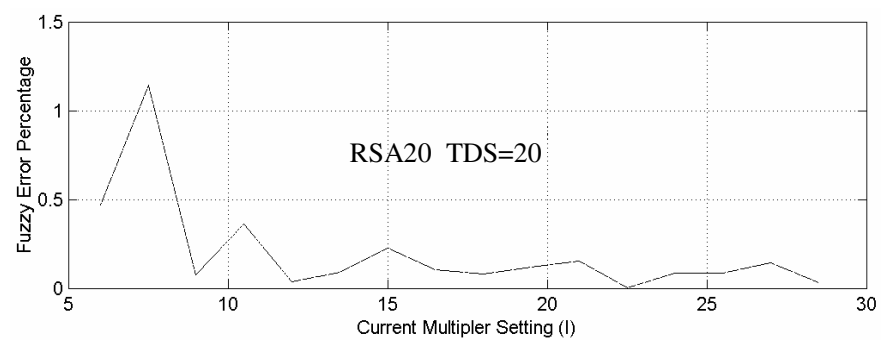

Fig. 5. Error percentage of fuzzy model for RSA20 when TDS=20

As can be seen from Fig. 4, the average of error percentages of the fuzzy model results are smaller than the polynomial form and in most cases is near 0.4 percent. In addition, Fig. 5 shows that the error percentage of the fuzzy model decreases when the fault current through the relay increases. This is an important feature, because high fault currents can cause more damages to the power systems components.

\subsubsection{Fuzzy Model of CRP9 OC Relay}

In this section, the fuzzy model is applied to CRP9 OC relay to find its characteristic. The relay is an electromechanical type and its data are given in Table 2. The data are obtained by experimental tests. The coefficients of the analytical model, i.e. $a_{0}$ to $a_{8}$ for the relay, are calculated and shown in Table 6.

Figures 6-7 show the obtained results after applying two different methods.

Table 6. Analytical polynomial coefficients for CRP9

\begin{tabular}{cccc}
\hline Coefficient & TDS $=4$ & TDS $=6$ & TDS $=10$ \\
\hline$a_{0}$ & 0.0769 & 0.1401 & 0.0757 \\
$a_{1}$ & 1.3063 & 0.1726 & 1.7057 \\
$a_{2}$ & -1.8269 & 5.262 & -3.8979 \\
$a_{3}$ & 4.7069 & -3.3488 & 7.9226 \\
$a_{4}$ & 6.6728 & -5.9993 & 11.2491 \\
$a_{5}$ & 1.3859 & -0.9672 & 1.0027 \\
$a_{6}$ & -7.1234 & 6.6096 & -15.5886 \\
$a_{7}$ & -15.6564 & 13.4513 & -32.4335 \\
$a_{8}$ & -22.6173 & 18.796 & -46.5238 \\
\hline
\end{tabular}

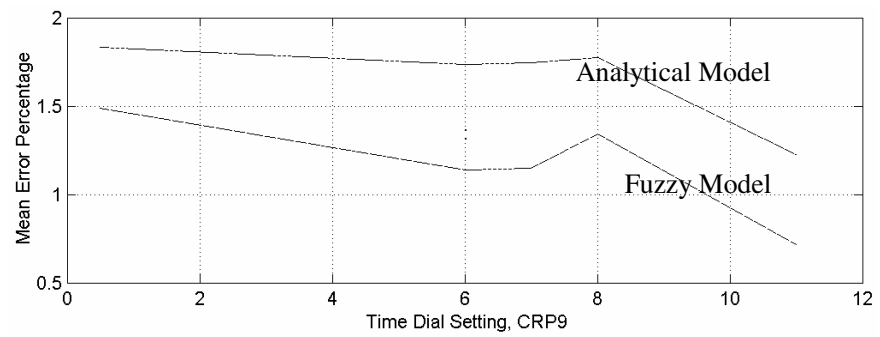

Fig. 6. Average error percentage of fuzzy and Analytical model for CRP9 


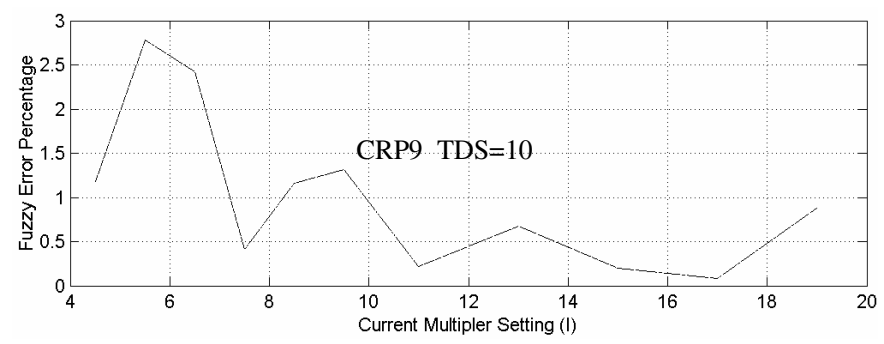

Fig. 7. Error percentage of fuzzy model for CRP9 when TDS=10

Figure 6 shows the fuzzy model of CRP9 is more precise than the analytical ninecoefficient model. Similar to RSA20, the average error percentage of proposed model is below 1.5 percent and usually decreases when current multiplier setting increases. On the other hand, the fuzzy method uses very simple mathematical equation and does not involve to complicated curve fitting techniques. Consequently, ill-structure matrices and poor results are not reported.

\section{Conclusion}

In this paper a new model for OC relays, based on fuzzy logic is presented. The new model was evaluated by experimental tests on two types of OC relays. The results revealed that the error percentages of fuzzy model for both relays were low. In comparing, the results of the new method with the analytical model, it was evident that the new model did not need any curve fitting technique. It has been shown that the method is flexible and can take into account different relay characteristics with linear and nonlinear features.

\section{Glossary}

$t$ : Operation time of OC relay

I: $\quad$ Current multiplier setting

$s: \quad$ Slope between two neighboured points on TC curve

$r: \quad$ Fuzzy correction factor

$\mu_{r}: \quad$ Membership function of $r$

$\mu_{s}: \quad$ Membership function of $\mathrm{s}$

$\mu_{I} \quad$ Membership function of $I$

$f: \quad$ Asymetric activation function

$y: \quad$ Internal activity of neuron

$a, b: \quad$ Constants

$x$ : $\quad$ TDS or TMS 


\section{Refrences}

1. T.S. Sidhu, M. hfuda and M.S. Sachdev, A technique for generating computer models of microprocessor-based relays, Proc. IEEE Communications, Power and Computing, WESCANEX'97, 1997, pp. 191-196.

2. P.G. McLaren, K. Mustaohi, G. Benmouyal, S. Chano, A. Giris, C. Henville, M. Kezunovic, L. Kojovic, R. Marttila, M. Meisinger, G. Michel, M.S. Sachdev, V. Skendzic, T.S. Sidue and D. Tziouvaras, Software models for relays, IEEE Trans. Power Delivery, 16(2001), 238-245.

3. M. S. Sachdev and T. S. Sidhu, Modelling relays for use in power system protection studies, Proc. IEE Development in Power System Protection, 2001, pp. 523-526.

4. IEEE Committee Report, Computer representation of overcurrent relay characteristics, IEEE Trans Power Deliver, 4(1989), 1659-1667.

5. IEEE Standard inverse-time characteristic equations for overcurrent relays, IEEE Std C37.112-1996.

6. H. Askarian, K. Faez and H. Kazemi, A new method for overcurrent relay (OC) using neural network and fuzzy logic, Proc IEEE Speech and Image Technologies for Telecommunications, TENCON '97, 1997, pp. 407-410.

7. H.T. Nguyen and E.A. Walker, A First Course in Fuzzy Logic, Chapman and Hall/CRC, New York, 2000, pp. 193 194.

8. L. Medsker and J. Liebowitz, Design and Development of Expert system and Neural networks, Macmillan, New York, 1994, pp.196 203.

9. L. Fausett, Fundamentals of Neural Networks, Architectures, Algorithms and Applications, Prentice-Hall, NJ, 1994, pp. 86 87.

10. S.S. Haykin, Neural Networks (A comprehensive foundation), Macmillan, New York, 1994, pp. 176-181.

11. T.F. Coleman and Y. Li , An interior, trust region approach for non-linear minimization subject to bounds, SIAM journal on Optimisation, 6(1996), 418-445.

12. T.F. Coleman and Y. Li, On the convergence of reflective Newton methods for large-scale nonlinear minimization subject to bounds, Mathematical Programming, 67(1994), 189224. 